\title{
ASYMPTOTIC ANALYSIS FOR PORTFOLIO OPTIMIZATION PROBLEM UNDER AN EXTENDED HESTON'S STOCHASTIC VOLATILITY MODEL
}

\author{
JI-HUN YOON ${ }^{1}$ AND SOTHEARA VENG ${ }^{2}$ \\ ${ }^{1,2}$ Department of Mathematics \\ Pusan National University \\ Pusan, 46241, REPUBLIC OF KOREA
}

\begin{abstract}
In this paper, we study the portfolio optimization problem under an extended Heston stochastic volatility model. By using asymptotic analysis technique, we are able to derive approximations of the optimal value function and the optimal strategy. We give an explicit asymptotic approximation of the optimal strategy for the case of hyperbolic absolute risk aversion utility functions and prove that the leading order term of the optimal strategy recovers the approximation up to the first order of the optimal value function.
\end{abstract}

AMS Subject Classification: 90C39, 90C59, 91G10, 35Q93

Key Words: portfolio optimization, Heston's model, stochastic volatility, asymptotic analysis, HARA utility

Received: $\quad$ May 1, 2017; Accepted: February 13, 2018;

Published: April 3, 2018 doi: 10.12732/dsa.v27i2.8

Dynamic Publishers, Inc., Acad. Publishers, Ltd. https://acadsol.eu/dsa

\section{INTRODUCTION}

The portfolio optimization problem was originally introduced and studied in continuoustime setting in the seminal papers by Merton, [6] and [7], where an investor has to choose how to invest between stocks and a money market account so that the expected utility of investment wealth is maximized. The problem is known to have explicit solutions when the stocks are assumed to follow the Black-Scholes model with constant returns and constant volatilities and the utility function modeling the 
investors preference is of certain types, for example, Constant Relative Risk Aversion (CRRA). Based on these pioneering papers, several authors have introduced more realistic and complicated market models. Typical examples are stochastic volatility models. In these models, the assumption of constant returns and constant volatilities of the Black-Scholes model has been relaxed by allowing the returns and the volatilities of underlying assets to evolve stochastically over time. [8] considered a one-factor stochastic volatility model where the drift and volatility terms of the asset price is driven by a diffusion process correlated with the geometric Brownian motion. Using a viscosity solution technique, she was able to derive explicit expressions for the optimal value function and for the optimal investment strategy under an appropriate assumption of the model parameters. [5] studied the case of the Heston stochastic volatility model and derived a closed-form solution for the CRRA utility function under the assumption that the market price of risk is a linear function of the volatility. [2] provided an explicit solution within a particular one-factor stochastic volatility model. A recent paper [4] considered the portfolio optimization problem under an extended Black-Scholes model where the coefficients of the risky asset are driven by two factors, one of which is fast mean-reverting and the other is slow varying. There, approximate solutions to the optimal value function and the optimal strategy for general utility functions were derived by using asymptotic analysis techniques. Specifically, with a combination of singular and regular perturbations with respect to the small parameters characterizing the two factors, they showed that the optimal value function and the optimal strategy could be approximated by the Merton formula plus the correction terms given in terms of derivatives of the Merton formula itself. In this study, we extend the results of [5] by adding a fast mean reverting factor to the Heston model and construct approximate solutions by using asymptotic analysis developed in [4]. We suppose that an investor manages his or her initial wealth by investing in a financial market consisting of a risky asset and a risk-free asset. The price $B_{t}$ of the risk-free asset at time $t$ follows the ordinary differential equation (ODE):

$$
d B_{t}=r B_{t} d t
$$

where $r>0$ is a constant interest rate. The price $S_{t}$ at time $t$ of the risky asset is described by the following stochastic differential equations (SDEs):

$$
\begin{aligned}
d S_{t} & =\mu\left(Y_{t}, Z_{t}\right) S_{t} d t+f\left(Y_{t}\right) \sqrt{Z_{t}} S_{t} d W_{t}^{s}, \\
d Y_{t} & =\frac{Z_{t}}{\epsilon} \beta\left(Y_{t}\right) d t+\sqrt{\frac{Z_{t}}{\epsilon}} \alpha\left(Y_{t}\right) d W_{t}^{y}, \\
d Z_{t} & =\kappa\left(\theta-Z_{t}\right) d t+\sigma \sqrt{Z_{t}} d W_{t}^{z},
\end{aligned}
$$


where $W^{s}, W^{y}$ and $W^{z}$ are correlated Brownian motions in a filtered probability space $\left(\Omega, \mathcal{F}, \mathcal{F}_{t}, P\right)$ with correlation structure given by

$$
\begin{aligned}
& d\left\langle W^{s}, W^{y}\right\rangle_{t}=\rho_{s y} d t \\
& d\left\langle W^{s}, W^{z}\right\rangle_{t}=\rho_{s z} d t, \\
& d\left\langle W^{y}, W^{z}\right\rangle_{t}=\rho_{y z} d t .
\end{aligned}
$$

The correlation coefficients $\rho_{s y}, \rho_{s z}$ and $\rho_{y z}$ are constants in $(-1,1)$ satisfying $\rho_{s y}^{2}+$ $\rho_{s z}^{2}+\rho_{y z}^{2}-2 \rho_{s y} \rho_{s z} \rho_{y z}<1$, so that the covariance matrix of the Brownian motions is guaranteed to be positive definite. Here, $f\left(Y_{t}\right) \sqrt{Z_{t}}$ is the volatility of the underlying asset driven by two diffusion processes $Y_{t}$ and $Z_{t}$. The process $Z_{t}$ is a Cox-IngersollRoss (CIR) process, where $\theta$ is the long run mean, $\kappa$ is the rate of mean reversion, and $\sigma$ is the the volatility of volatility. The parameters $\kappa, \theta$ and $\sigma$ are positive constants and required to satisfy the Feller condition $2 \kappa \theta \geq \sigma^{2}$ to ensure that the process $Z_{t}$ is always positive starting with $Z_{0}>0$. We will specify assumptions on the coefficients $\mu(y, z), f(y), \alpha(y)$ and $\beta(y)$ of our model later.

We assume the process $Y_{t}$ given in (3) satisfies

$$
Y_{t} \stackrel{d}{=} Y_{\frac{z_{t}}{\epsilon} t}^{(1)}
$$

where the notation $\stackrel{d}{=}$ means equality in distribution and $Y_{t}^{(1)}$ is an ergodic diffusion process with unique invariant distribution denoted by $\Phi$. Then, by its construction in (3), $Y_{t}$ also has unique invariant distribution $\Phi$ and is a fast mean-reverting process when the parameter $\epsilon$ is small. We denote the average with respect to $\Phi$ for a measurable function $g$ by

$$
\langle g\rangle=\int g(y) \Phi(d y) .
$$

We observe that in the case that $f(y)=1$ and $\mu(y, z)=\mu(z)$ is independent of $y$, our model reduces the Heston stochastic volatility model considered by [5]. Note also that when $\beta(y)=m-y$ and $\alpha(y)=\sqrt{2} \nu$, with $m$ and $\nu$ constant, the model becomes the generalized Heston model considered in [3] for an option pricing problem. So, we may call the stochastic dynamic system (2)-(4) the extended Heston stochastic volatility model.

At time $t \in[0, T]$, the investor allocates the fraction $\pi_{t}$ of the wealth in the risky asset and the rest is invested in the risk-free asset. Then, the wealth process $X_{t}$ associated with $\pi(\cdot)$ satisfies the following SDE:

$$
d X_{t}=X_{t}\left(r+\pi_{t}\left(\mu\left(Y_{t}, Z_{t}\right)-r\right)\right) d t+\pi_{t} f\left(Y_{t}\right) \sqrt{Z_{t}} X_{t} d W_{t}^{s},
$$

with initial wealth $X_{0}=x>0$. We assume that all coefficients of the above SDEs are $\mathcal{F}_{t}$-progressively measurable and that the SDEs (2)-(4) and (6) have unique strong 
solutions. As demonstrated in [5], existence and uniqueness of the CIR process $Z_{t}$ in (4) is ensured by the so-called Yamada and Watanabe conditions. Also, the conditions

$$
\int_{0}^{T}\left|\mu\left(Y_{t}, Z_{t}\right)\right| d t+\mathbb{E}\left[\int_{0}^{T}\left|f^{2}\left(Y_{t}\right) Z_{t}\right| d t\right]<\infty
$$

and

$$
\int_{0}^{T}\left|\pi_{t}\left(\mu\left(Y_{t}, Z_{t}\right)-r\right)\right| d t+\mathbb{E}\left[\int_{0}^{T}\left|\pi_{t}^{2} f^{2}\left(Y_{t}\right) Z_{t}\right| d t\right]<\infty
$$

guarantee existence and uniqueness of strong solutions for (2) and (6), respectively. The control function $\pi(\cdot)$ is said to be admissible if it is $\mathcal{F}_{t}$-progressively measurable, satisfies

$$
\mathbb{E}\left[\int_{0}^{T} \pi_{t}^{2} f^{2}\left(Y_{t}\right) Z_{t} X_{t}^{2} d t\right]<\infty
$$

and is such that the above assumption for all processes is satisfied. We denote the set of all admissible strategies by $\mathcal{A}$.

As in [5], we assume an unbounded market price of risk in our model. More specifically, assuming that $\mu\left(Y_{t}, Z_{t}\right)-r=\mu_{0}\left(Y_{t}\right) Z_{t}$, the market price of risk $\zeta_{t}$ is of the form

$$
\zeta_{t}=\frac{\mu\left(Y_{t}, Z_{t}\right)-r}{f\left(Y_{t}\right) \sqrt{Z_{t}}}=\frac{\mu_{0}\left(Y_{t}\right)}{f\left(Y_{t}\right)} \sqrt{Z_{t}}:=\lambda\left(Y_{t}\right) \sqrt{Z_{t}},
$$

where $\lambda(y)$ is a function that is bounded and bounded away from zero. The particular form of $f(y)$ does not play an important role in our asymptotic results derived later. So, we just assume that it is bounded away from zero, that is, there exists a constant $l$ such that $|f(y)| \geq l>0$.

The main contribution of the present work is that we can treat the portfolio optimization within the above extended Heston model for the hyperbolic absolute risk aversion (HARA) utility functions that include the CRRA and the constant absolute risk aversion (CARA) types. As our market model can be viewed as a fast meanreverting correction to the Heston model, the optimization problem will be solved by using a singular perturbation method. The resulting asymptotic approximations to the optimal value function and the optimal strategy are obtained in closed-form, which is our main result extending the study of [5].

The remaining structure of this paper is as follows. In Section 2, we formulate our portfolio optimization problem and derive the associated HJB equation. In Section 3, we employ asymptotic analysis method to obtain an explicit approximation to the optimal value function for the HARA utility functions. In Section 4, we use the asymptotic approximation for the optimal value function derived in Section 3 to compute the first order correction to the optimal investment strategy, which consists of the leading order and the first order correction terms. And we show that the leading order term of the optimal strategy can recover the optimal value function up to 
the first order correction. Section 5 concludes and suggests future research plans. In Appendix, we review some background results on the Heston model and give the proofs to all theorems.

\section{MODEL FORMULATION}

In our market model, the investor is assumed to have a utility function $U(x)$ of the HARA type, which includes power, exponential and logarithm utility functions. For a fixed parameter $\epsilon$, we denote by $X_{t}^{\epsilon, \pi}, t \in[0, T]$ the solution of (6) corresponding to the strategy $\pi(\cdot)$.

The objective of the investor is to maximize the expected utility from terminal wealth

$$
\sup _{\pi \in \mathcal{A}} \mathbb{E}\left[U\left(X_{T}^{\pi}\right)\right]
$$

where $U(x)$ is the HARA utility function defined by

$$
U(x ; p, q, \eta)=\frac{1-p}{p q}\left(\frac{q x}{1-p}+\eta\right)^{p}
$$

with $q>0, p<1, p \neq 1$ and $\frac{q x}{1-p}+\eta>0$. To this end, we employ stochastic dynamic programming approach (cf.[1]). We begin by defining the value function corresponding to the strategy $\pi(\cdot)$ by

$$
V^{\epsilon, \pi}(t, x, y, z)=\mathbb{E}\left[U\left(X_{T}^{\pi}\right) \mid X_{t}^{\pi}=x, Y_{t}=y, Z_{t}=z\right]
$$

for all $(t, x, y, z) \in[0, T] \times \mathbb{R}^{3}$. Here, $\mathbb{E}[X \mid A]$ is the conditional expectation of a random variable $X$ given an event $A$. Then, we define the optimal value function $V^{\epsilon}(t, x, y, z)$ by

$$
V^{\epsilon}(t, x, y, z)=\sup _{\pi \in \mathcal{A}} \mathbb{E}\left[U\left(X_{T}^{\pi}\right) \mid X_{t}^{\pi}=x, Y_{t}=y, Z_{t}=z\right]
$$

Therefore, the associated Hamilton-Jacobi-Bellman (HJB) equation for $V^{\epsilon}$ is given by

$$
\begin{array}{r}
V_{t}^{\epsilon}+\frac{z}{\epsilon} \mathcal{L}_{0} V^{\epsilon}+r x V_{x}^{\epsilon}+\kappa(\theta-z) V_{z}^{\epsilon}+\frac{1}{2} \sigma^{2} z V_{z z}^{\epsilon} \\
+\frac{1}{\sqrt{\epsilon}} \rho_{y z} \sigma \alpha(y) z V_{y z}^{\epsilon}+\sup _{\pi}\left[\frac{1}{2} \pi^{2} f^{2}(y) z x^{2} V_{x x}^{\epsilon}\right. \\
\left.+\pi z x\left(\mu_{0}(y) V_{x}^{\epsilon}+\rho_{s z} \sigma f(y) V_{x z}^{\epsilon}+\frac{1}{\sqrt{\epsilon}} \rho_{s y} \alpha(y) f(y) V_{x y}^{\epsilon}\right)\right]=0,
\end{array}
$$

for $t \in[0, T), x \in \mathbb{R}^{+}, y \in \mathbb{R}$ and $z \in \mathbb{R}^{+}$, with the terminal condition

$$
V^{\epsilon}(T, x, y, z)=U(x)
$$


Here, $\mathcal{L}_{0}$ is the infinitesimal operator of the process $Y^{(1)}$ defined by

$$
\mathcal{L}_{0}=\frac{1}{2} \alpha^{2}(y) \frac{\partial^{2}}{\partial y^{2}}+\beta(y) \frac{\partial}{\partial y} .
$$

As $\pi$ appears in (10) as a quadratic expression, the maximizer, known as the optimal strategy, is given in the following feedback form:

$$
\pi^{*}(t, x, y, z)=-\frac{\lambda(y) V_{x}^{\epsilon}+\rho_{s z} \sigma V_{x z}^{\epsilon}+\frac{1}{\sqrt{\epsilon}} \rho_{s y} \alpha(y) V_{x y}^{\epsilon}}{f(y) x V_{x x}^{\epsilon}} .
$$

Substituting this optimal strategy into (10) yields

$$
\begin{array}{r}
V_{t}^{\epsilon}+\frac{z}{\epsilon} \mathcal{L}_{0} V^{\epsilon}+r x V_{x}^{\epsilon}+\kappa(\theta-z) V_{z}^{\epsilon}+\frac{1}{2} \sigma^{2} z V_{z z}^{\epsilon}+\frac{1}{\sqrt{\epsilon}} \rho_{y z} \sigma \alpha(y) z V_{y z}^{\epsilon} \\
-\frac{z\left(\lambda(y) V_{x}^{\epsilon}+\rho_{s z} \sigma V_{x z}^{\epsilon}+\frac{1}{\sqrt{\epsilon}} \rho_{s y} \alpha(y) V_{x y}^{\epsilon}\right)^{2}}{2 V_{x x}^{\epsilon}}=0 .
\end{array}
$$

Assumption 1. We assume that the optimal value function $V^{\epsilon}(t, x, y, z)$ is strictly increasing, strictly concave in $x$ for each $t \in[0, T), y \in \mathbb{R}$ and $z \in \mathbb{R}^{+}$, and is sufficiently smooth on the domain $[0, T] \times \mathbb{R}^{+} \times \mathbb{R} \times \mathbb{R}^{+}$. We also assume that it is the unique solution for the HJB equation (10) with terminal condition (11).

We note that for small $\epsilon$ the fully nonlinear PDE (14) is a singular perturbation around the portfolio optimization problem under the Heston model with an unbounded market price of risk considered in [5]. Hence, it is difficult to obtain the optimal value function either analytically or numerically in general setting. Instead, we will employ an asymptotic analysis as developed in [4] to construct an approximate solution as shown in the following section.

\section{ASYMPTOTIC ANALYSIS}

We now perform asymptotic analysis as developed in [4] to obtain an approximation to the value function satisfying (14) with terminal condition (11). We begin by expanding the optimal value function in powers of $\sqrt{\epsilon}$ :

$$
V^{\epsilon}=V^{(0)}+\sqrt{\epsilon} V^{(1)}+\epsilon V^{(2)}+\cdots
$$

Substituting the expansion (15) into (14) and grouping terms in successive powers of $\epsilon$, at the terms in $\epsilon^{-1}$ we have

$$
z \mathcal{L}_{0} V^{(0)}-\frac{1}{2} \rho_{s y}^{2} \alpha^{2}(y) z \frac{\left(V_{x y}^{(0)}\right)^{2}}{V_{x x}^{(0)}}=0 .
$$


Since all terms of the operator $\mathcal{L}_{0}$ in $(12)$ take derivatives in $y$, we choose $V^{(0)}$ to be independent of $y$ so that the equation (16) is satisfied. It follows from this choice that $V_{y}^{(0)}=0$, and then the expansion of (14) up to the order $\sqrt{\epsilon}$ is given by

$$
\begin{array}{r}
V_{t}^{(0)}+\sqrt{\epsilon} V_{t}^{(1)}+z \mathcal{L}_{0}\left(\frac{1}{\sqrt{\epsilon}} V^{(1)}+V^{(2)}+\sqrt{\epsilon} V^{(3)}\right) \\
+r x\left(V_{x}^{(0)}+\sqrt{\epsilon} V_{x}^{(1)}\right)+\kappa(\theta-z)\left(V_{z}^{(0)}+\sqrt{\epsilon} V_{z}^{(1)}\right) \\
+\frac{1}{2} \sigma^{2} z\left(V_{z z}^{(0)}+\sqrt{\epsilon} V_{z z}^{(1)}\right)+\rho_{y z} \sigma \alpha(y) z\left(V_{y z}^{(1)}+\sqrt{\epsilon} V_{y z}^{(2)}\right) \\
-z\left(\lambda(y)\left(V_{x}^{(0)}+\sqrt{\epsilon} V_{x}^{(1)}\right)+\rho_{s z} \sigma\left(V_{x z}^{(0)}+\sqrt{\epsilon} V_{x z}^{(1)}\right)\right. \\
\left.+\rho_{s y} \alpha(y)\left(V_{x y}^{(1)}+\sqrt{\epsilon} V_{x y}^{(2)}\right)\right)^{2} \frac{1}{2 V_{x x}^{(0)}}\left(1-\sqrt{\epsilon} \frac{V_{x x}^{(1)}}{V_{x x}^{(0)}}\right)+\ldots=0,
\end{array}
$$

Hence, we see from (17) that the only one term in $\epsilon^{-\frac{1}{2}}$ leads to

$$
\mathcal{L}_{0} V^{(1)}=0
$$

By the definition of $\mathcal{L}_{0}, V^{(1)}$ must be independent of $y$ (otherwise, $V^{(1)}$ would grow as much as $e^{y^{2} / 2}$ as $\left.y \rightarrow \infty\right)$. Using the fact that $V^{(0)}$ and $V^{(1)}$ are independent of $y$, the constant terms in (17) lead to

$$
\begin{array}{r}
V_{t}^{(0)}+z \mathcal{L}_{0} V^{(2)}+r x V_{x}^{(0)}+\kappa(\theta-z) V_{z}^{(0)}+\frac{1}{2} \sigma^{2} z V_{z z}^{(0)} \\
-\frac{z\left(\lambda(y) V_{x}^{(0)}+\rho_{s z} \sigma V_{x z}^{(0)}\right)^{2}}{2 V_{x x}^{(0)}}=0 .
\end{array}
$$

Viewing (19) as a Poisson equation for $V^{(2)}$ in $y$, the centering condition on the source term is given by

$$
\begin{array}{r}
\left\langle V_{t}^{(0)}+r x V_{x}^{(0)}+\kappa(\theta-z) V_{z}^{(0)}+\frac{1}{2} \sigma^{2} z V_{z z}^{(0)}\right. \\
\left.-\frac{z\left(\lambda(y) V_{x}^{(0)}+\rho_{s z} \sigma V_{x z}^{(0)}\right)^{2}}{2 V_{x x}^{(0)}}\right\rangle=0,
\end{array}
$$

where $\langle\cdot\rangle$ is the averaging operator defined in (5). Then it follows that

$$
\begin{aligned}
V_{t}^{(0)}+r x V_{x}^{(0)}+ & \kappa(\theta-z) V_{z}^{(0)}+\frac{1}{2} \sigma^{2} z V_{z z}^{(0)}-\frac{1}{2} \tilde{\lambda}^{2} z \frac{\left(V_{x}^{(0)}\right)^{2}}{V_{x x}^{(0)}} \\
& -\rho_{s z} \sigma \bar{\lambda} z \frac{V_{x}^{(0)} V_{z x}^{0}}{V_{x x}^{(0)}}-\frac{1}{2} \rho_{s z}^{2} \sigma^{2} z \frac{\left(V_{z x}^{(0)}\right)^{2}}{V_{x x}^{(0)}}=0,
\end{aligned}
$$


where we used the fact that $V^{(0)}$ is independent of $y$ and the notation $\bar{\lambda}=\langle\lambda\rangle$ and $\tilde{\lambda}=\sqrt{\left\langle\lambda^{2}\right\rangle}$. From (15), the terminal condition for (21) is given as

$$
V^{(0)}(T, x, z)=U(x)
$$

Observe that when $\lambda(y)=\lambda$, a constant, the nonlinear PDE (21) is the HJB equation corresponding to the Heston model studied by [5], where its solution is reviewed in Appendix A. There, explicit solutions for the HARA utility functions (including the power and the exponential utility functions) are derived. We recall that [5] derived explicit solutions for only the power utility function.

The following theorem contains the explicit formula of $V^{(0)}$ that satisfies the PDE (21) with terminal condition (22) for the HARA utility function, where its proof is given in a similar way as in Appendix A.

Theorem 2. For the HARA utility function $U(x)$ given in (8), the PDE (21) with the terminal condition (22) has an explicit solution

$$
V^{(0)}(t, x, z)=\frac{1-p}{p q}\left(\frac{q}{1-p} x e^{r(T-t)}+\eta\right)^{p} e^{A(t)+B(t) z},
$$

where the functions $A(t)$ and $B(t)$ are given as in the following cases:

- Case 1: $\Delta>0$.

$$
\begin{aligned}
A(t)= & \frac{\kappa \theta}{\sigma^{2}\left(1+\Gamma \rho_{s z}^{2}\right)}\left(\left(\kappa-\Gamma \rho_{s z} \sigma \bar{\lambda}+\sqrt{\Delta}\right)(T-t)\right. \\
& \left.-2 \ln \left(\frac{1-g e^{(T-t) \sqrt{\Delta}}}{1-g}\right)\right), \\
B(t)= & \frac{\kappa-\Gamma \rho_{s z} \sigma \bar{\lambda}+\sqrt{\Delta}}{\sigma^{2}\left(1+\Gamma \rho_{s z}^{2}\right)}\left(\frac{1-e^{\sqrt{\Delta}(T-t)}}{1-g e^{\sqrt{\Delta}(T-t)}}\right) .
\end{aligned}
$$

- Case 2: $\Delta=0$ and $T K+1>0$.

$$
\begin{aligned}
A(t)= & \frac{\kappa \theta}{\sigma^{2}\left(1+\Gamma \rho_{s z}^{2}\right)}(2 K(T-t) \\
& -2 \ln (1+K(T-t))), \\
B(t)= & \frac{2 K^{2}}{\sigma^{2}\left(1+\Gamma \rho_{s z}^{2}\right)}\left(\frac{T-t}{1+K(T-t)}\right) .
\end{aligned}
$$

Here, we define $\Delta, g, K$ and $\Gamma$ by

$$
\Delta=\kappa^{2}-\Gamma\left(2 \kappa \rho_{s z} \sigma \bar{\lambda}+\sigma^{2} \tilde{\lambda}^{2}\right)+\Gamma^{2} \rho_{s z}^{2} \sigma^{2}\left(\bar{\lambda}^{2}-\tilde{\lambda}^{2}\right),
$$




$$
\begin{aligned}
g & =\frac{\kappa-\Gamma \rho_{s z} \sigma \bar{\lambda}+\sqrt{\Delta}}{\kappa-\Gamma \rho_{s z} \sigma \bar{\lambda}-\sqrt{\Delta}}, \\
K & =\frac{1}{2}\left(\kappa-\Gamma \rho_{s z} \sigma \bar{\lambda}\right), \\
\Gamma & =\frac{p}{1-p} .
\end{aligned}
$$

Remark 3. [(1).]

1. We can easily see from $(23)$ that $V^{(0)}$ satisfies

$$
V_{z}^{(0)}(t, x, z)=B(t) V^{(0)}(t, x, z)
$$

for all $(t, x, z) \in[0, T] \times \mathbb{R}^{+} \times \mathbb{R}^{+}$. This relation leads to the possibility to explicitly derive the first order correction term $V^{(1)}$ in terms of $V^{(0)}$, which is the main result of this paper.

2. From $(23)$, we observe that the ratio $\frac{V_{x}^{(0)}}{V_{x x}^{(0)}}$ does not depend on $z$, and so we denote

$$
R(t, x)=-\frac{V_{x}^{(0)}(t, x, z)}{V_{x x}^{(0)}(t, x, z)} .
$$

Before continuing our asymptotic analysis, we define the differential operators $D_{j}$ by

$$
D_{j}=R^{j}(t, x) \frac{\partial^{j}}{\partial x^{j}}, \quad j=1,2, \ldots,
$$

and the linear operator $\mathcal{L}_{t, x, z}\left(\lambda_{1}, \lambda_{2}\right)$ by

$$
\begin{array}{r}
\mathcal{L}_{t, x, z}\left(\lambda_{1}, \lambda_{2}\right)=\frac{\partial}{\partial t}+ \\
+x \frac{\partial}{\partial x}+\kappa(\theta-z) \frac{\partial}{\partial z}+\frac{1}{2} \sigma^{2} z \frac{\partial^{2}}{\partial z^{2}} \\
+\left(\lambda_{1}^{2}+\rho_{s z} \sigma \lambda_{2} B(t)\right) z D_{1} \\
+\frac{1}{2}\left(\lambda_{1}^{2}+2 \rho_{s z} \sigma \lambda_{2} B(t)+\rho_{s z}^{2} \sigma^{2} B^{2}(t)\right) z D_{2} \\
+\rho_{s z} \sigma\left(\lambda_{2}+\rho_{s z} \sigma B(t)\right) z D_{1} \frac{\partial}{\partial z}
\end{array}
$$

Then, by using of (29) direct computation shows that the equation (21) can be written as

$$
\mathcal{L}_{t, x, z}(\tilde{\lambda}, \bar{\lambda}) V^{(0)}=0 .
$$

Similarly, we can rewrite (19) as

$$
z \mathcal{L}_{0} V^{(2)}+\mathcal{L}_{t, x, z}(\lambda(y), \lambda(y)) V^{(0)}=0 .
$$

Then it follows from (33) and (34) that

$$
\mathcal{L}_{0} V^{(2)}=-\frac{1}{z}\left(\mathcal{L}_{t, x, z}(\lambda(y), \lambda(y))-\mathcal{L}_{t, x, z}(\tilde{\lambda}, \bar{\lambda})\right) V^{(0)} .
$$


Hence, up to a constant in $y$, we choose

$$
V^{(2)}=-\frac{1}{z} \mathcal{L}_{0}^{-1}\left(\mathcal{L}_{t, x, z}(\lambda(y), \lambda(y))-\mathcal{L}_{t, x, z}(\tilde{\lambda}, \bar{\lambda})\right) V^{(0)}
$$

where $\mathcal{L}_{0}^{-1}$ is the inverse operator of $\mathcal{L}_{0}$.

Now, we proceed asymptotic analysis to derive the first order term $V^{(1)}$. By using (29), the terms in $\sqrt{\epsilon}$ of the expanded PDE (17) lead to

$$
\begin{array}{r}
z \mathcal{L}_{0} V^{(3)}+V_{t}^{(1)}+r x V_{x}^{(0)}+\kappa(\theta-z) V_{z}^{(1)}+\frac{1}{2} \sigma^{2} z V_{z z}^{(1)} \\
+\rho_{y z} \sigma \alpha(y) z V_{y z}^{(2)}+\frac{z}{2 V_{x x}^{(0)}}\left[\left(\lambda(y)+\rho_{s z} \sigma B(t)\right)^{2}\left(V_{x}^{(0)}\right)^{2} \frac{V_{x x}^{(1)}}{V_{x x}^{(0)}}\right. \\
-2\left(\lambda(y)+\rho_{s z} \sigma B(t)\right) V_{x}^{(0)}\left(\lambda(y) V_{x}^{(1)}+\rho_{s z} \sigma V_{x z}^{(1)}\right. \\
\left.\left.+\rho_{s y} \alpha(y) V_{x y}^{(2)}\right)\right]=0 .
\end{array}
$$

Then using (30) and (31), we can write (37) as

$$
z \mathcal{L}_{0} V^{(3)}+\mathcal{L}_{t, x, z}(\lambda(y), \lambda(y)) V^{(1)}+z \mathcal{L}_{x, y, z} V^{(2)}=0
$$

where the operator $\mathcal{L}_{x, y, z}$ is defined by

$$
\mathcal{L}_{x, y, z}=\rho_{y z} \sigma \alpha(y) \frac{\partial^{2}}{\partial y \partial z}+\rho_{s y} \alpha(y)\left(\lambda(y)+\rho_{s z} \sigma B(t)\right) R \frac{\partial^{2}}{\partial x \partial y} .
$$

Viewing (38) as a Poisson equation for $V^{(3)}$ in $y$, the centering condition requires that

$$
\left\langle\mathcal{L}_{t, x, z}(\lambda(y), \lambda(y)) V^{(1)}+z \mathcal{L}_{x, y, z} V^{(2)}\right\rangle=0 .
$$

Since $V^{(1)}$ does not depend on $y$, the equation above can written as

$$
\mathcal{L}_{t, x, z}(\tilde{\lambda}, \bar{\lambda}) V^{(1)}=-z\left\langle\mathcal{L}_{x, y, z} V^{(2)}\right\rangle .
$$

Substituting $V^{(2)}$, given by (36), into this equation yields

$$
\mathcal{L}_{t, x, z}(\tilde{\lambda}, \bar{\lambda}) V^{(1)}=\mathcal{A} V^{(0)}
$$

where $\mathcal{A}:=z\left\langle\mathcal{L}_{x, y, z} \frac{1}{z} \mathcal{L}_{0}^{-1}\left(\mathcal{L}_{t, x, z}(\lambda(y), \lambda(y))-\mathcal{L}_{t, x, z}(\tilde{\lambda}, \bar{\lambda})\right)\right\rangle$.

Now, we explicitly compute the source term of (41). To do this, we introduce two functions $\phi$ and $\psi$ that satisfy the following Poisson equations

$$
\begin{aligned}
\mathcal{L}_{0} \phi(y) & =\frac{1}{2}\left(\lambda^{2}(y)-\tilde{\lambda}^{2}\right) \\
\mathcal{L}_{0} \psi(y) & =\lambda(y)-\bar{\lambda}
\end{aligned}
$$


By observing from $(23)$ that $D_{1} V^{(0)}=\Gamma V^{(0)}$, where $\Gamma$ is defined in (28), we have

$$
\begin{aligned}
\mathcal{A} V^{(0)}= & z\left\langle\mathcal { L } _ { x , y , z } \frac { 1 } { z } \mathcal { L } _ { 0 } ^ { - 1 } \left(\frac{1}{2}\left(\lambda^{2}(y)-\tilde{\lambda}^{2}\right)\right.\right. \\
& \left.\left.+\rho_{s z} \sigma B(t)(\lambda(y)-\bar{\lambda})\right) z D_{1} V^{(0)}\right\rangle \\
= & z \Gamma\left\langle\mathcal{L}_{x, y, z} \phi(y) V^{(0)}\right\rangle+\rho_{s z} \sigma B(t) z \Gamma\left\langle\mathcal{L}_{x, y, z} \psi(y) V^{(0)}\right\rangle \\
= & z\left(\rho_{s y} \Gamma^{2} F_{3}+\sigma \Gamma\left(\rho_{y z} F_{1}+\rho_{s y} \rho_{s z} \Gamma\left(F_{1}+F_{4}\right)\right) B(t)\right. \\
& \left.+\rho_{s z} \sigma^{2} \Gamma\left(\rho_{y z}+\rho_{s y} \rho_{s z} \Gamma\right) F_{2} B^{2}(t)\right) V^{(0)},
\end{aligned}
$$

where the constants $F_{i}$ are defined by

$$
\begin{aligned}
& F_{1}=\left\langle\alpha \phi^{\prime}\right\rangle, \\
& F_{2}=\left\langle\alpha \psi^{\prime}\right\rangle, \\
& F_{3}=\left\langle\alpha \lambda \phi^{\prime}\right\rangle, \\
& F_{4}=\left\langle\alpha \lambda \psi^{\prime}\right\rangle .
\end{aligned}
$$

From the expansion (15), the PDE (41) has the terminal condition

$$
V^{(1)}(T, x, z)=0 \text {. }
$$

Up to now, we have shown that the first order term $V^{(1)}$ satisfies the linear PDE (41) with the terminal condition (49). In the following theorem, we derive an explicit expression for $V^{(1)}$ in terms of $V^{(0)}$ for the first time, which is the main result of this study.

Theorem 4. The linear PDE (41) with terminal condition (49) has a solution of the form

$$
V^{(1)}(t, x, z)=\left(\kappa \theta g_{1}(t)+g_{2}(t) z\right) V^{(0)}(t, x, z),
$$

where $V^{(0)}$ is given in Theorem 2, and $g_{1}(t)$ and $g_{2}(t)$ are defined in the following cases:

- Case 1: $\Delta>0$.

$$
\begin{aligned}
& g_{1}(t)=-\left(c_{0} J_{0}(t)+c_{1} J_{1}(t)+c_{2} J_{2}(t)\right) \\
& g_{2}(t)=-\left(c_{0} I_{0}(t)+c_{1} I_{1}(t)+c_{2} I_{2}(t)\right) \\
& I_{0}(t)=\frac{-1+\left(1-2 g \sqrt{\Delta}(T-t)-g^{2}\right) e^{\sqrt{\Delta}(T-t)}+g^{2} e^{2 \sqrt{\Delta}(T-t)}}{\sqrt{\Delta}\left(1-g e^{\sqrt{\Delta}(T-t)}\right)^{2}}, \\
& I_{1}(t)=\frac{-1+(1-(1+g) \sqrt{\Delta}(T-t)-g) e^{\sqrt{\Delta}(T-t)}+g e^{2 \sqrt{\Delta}(T-t)}}{\sqrt{\Delta}\left(1-g e^{\sqrt{\Delta}(T-t)}\right)^{2}},
\end{aligned}
$$




$$
\begin{aligned}
I_{2}(t)= & \frac{-1-2 \sqrt{\Delta}(T-t) e^{\sqrt{\Delta}(T-t)}+e^{2 \sqrt{\Delta}(T-t)}}{\sqrt{\Delta}\left(1-g e^{\sqrt{\Delta}(T-t)}\right)^{2}} \\
J_{0}(t)= & -\frac{1+g+\sqrt{\Delta}(T-t)-(1+g-\sqrt{\Delta} g(T-t)) e^{\sqrt{\Delta}(T-t)}}{\Delta\left(1-g e^{\sqrt{\Delta}(T-t)}\right)} \\
J_{1}(t)= & -\frac{2+\sqrt{\Delta}(T-t)-(2-\sqrt{\Delta}(T-t)) e^{\sqrt{\Delta}(T-t)}}{\Delta\left(1-g e^{\sqrt{\Delta}(T-t)}\right)} \\
J_{2}(t)= & -\frac{1+g+\sqrt{\Delta} g(T-t)-(1+g-(2-g) \sqrt{\Delta} g(T-t)) e^{\sqrt{\Delta}(T-t)}}{\Delta g\left(1-g e^{\sqrt{\Delta}(T-t)}\right)} \\
& +\frac{(1-g)^{2}}{\Delta g^{2}} \ln \left(\frac{1-g e^{\sqrt{\Delta}(T-t)}}{1-g}\right) \\
c_{0}= & \rho_{s y} \Gamma^{2} F_{3}, \\
c_{1}= & \sigma \Gamma\left(\rho_{y z} F_{1}+\rho_{s y} \rho_{s z} \Gamma\left(F_{1}+F_{4}\right)\right)\left(\frac{\kappa-\Gamma \rho_{s z} \sigma \bar{\lambda}+\sqrt{\Delta}}{\sigma^{2}\left(1+\Gamma \rho_{s z}^{2}\right)}\right) \\
c_{2}= & \rho_{s z} \sigma^{2} \Gamma\left(\rho_{y z}+\rho_{s y} \rho_{s z} \Gamma\right) F_{2}\left(\frac{\kappa-\Gamma \rho_{s z} \sigma \bar{\lambda}+\sqrt{\Delta}}{\sigma^{2}\left(1+\Gamma \rho_{s z}^{2}\right)}\right)^{2}
\end{aligned}
$$

- Case 2: $\Delta=0$ and $T K+1>0$.

$$
\begin{aligned}
g_{1}(t) & =-\left(\bar{c}_{0} \bar{J}_{0}(t)+\bar{c}_{1} \bar{J}_{1}(t)+\bar{c}_{2} \bar{J}_{2}(t)\right) \\
g_{2}(t) & =-\left(\bar{c}_{0} \bar{I}_{0}(t)+\bar{c}_{1} \bar{I}_{1}(t)+\bar{c}_{2} \bar{I}_{2}(t)\right) \\
\bar{I}_{0}(t) & =\frac{1}{(1+K(T-t))^{2}}\left((T-t)+K(T-t)^{2}+\frac{K^{2}}{3}(T-t)^{3}\right) \\
\bar{I}_{1}(t) & =\frac{1}{(1+K(T-t))^{2}}\left(\frac{1}{2}(T-t)^{2}+\frac{K}{3}(T-t)^{3}\right) \\
\bar{I}_{2}(t) & =\frac{(T-t)^{3}}{3(1+K(T-t))^{2}} \\
\bar{J}_{0}(t) & =\frac{(T-t)^{2}(3+K(T-t))}{6(1+K(T-t))} \\
\bar{J}_{1}(t) & =\frac{(T-t)^{3}}{6(1+K(T-t))} \\
\bar{J}_{2}(t) & =\frac{-6(T-t)-3 K(T-t)^{2}+K^{2}(T-t)^{3}}{6 K^{3}(1+K(T-t))}+\frac{1}{K^{4}} \ln (1+K(T-t)) \\
\bar{c}_{0} & =\rho_{s y} \Gamma^{2} F_{3}
\end{aligned}
$$




$$
\begin{aligned}
& \bar{c}_{1}=\sigma \Gamma\left(\rho_{y z} F_{1}+\rho_{s y} \rho_{s z} \Gamma\left(F_{1}+F_{4}\right)\right) \frac{\left(\kappa-\Gamma \rho_{s z} \sigma \bar{\lambda}\right)^{2}}{2 \sigma^{2}\left(1+\Gamma \rho_{s z}^{2}\right)}, \\
& \bar{c}_{2}=\rho_{s z} \sigma^{2} \Gamma\left(\rho_{y z}+\rho_{s y} \rho_{s z} \Gamma\right) F_{2}\left(\frac{\left(\kappa-\Gamma \rho_{s z} \sigma \bar{\lambda}\right)^{2}}{2 \sigma^{2}\left(1+\Gamma \rho_{s z}^{2}\right)}\right)^{2} .
\end{aligned}
$$

Here, $F_{1}, F_{2}, F_{3}$ and $F_{4}$ are given by (45),(46),(47) and (48), respectively. $\Delta, g, K$ and $\Gamma$ are defined as in Theorem 2.

Proof. See Appendix B.

\section{OPTIMAL STRATEGY}

Since we have derived the first two terms $V^{(0)}$ and $V^{(1)}$ for the optimal value function in the previous section, we can proceed to derive an asymptotic approximation to the optimal strategy $\pi^{*}$ given by (13). Like the case of the optimal value function, we look for the optimal strategy $\pi^{*}$ of the form

$$
\pi^{*}=\pi^{*(0)}+\sqrt{\epsilon} \pi^{*(1)}+\epsilon \pi^{*(2)}+\cdots
$$

and only the expressions for $\pi^{*(0)}$ and $\pi^{*(1)}$ will be derived.

Substituting the expansion (15) for $V^{\epsilon}$ into (13) gives

$$
\begin{aligned}
\pi^{*}= & -\left[\lambda(y)\left(V_{x}^{(0)}+\sqrt{\epsilon} V_{x}^{(1)}\right)+\rho_{s z} \sigma\left(V_{x z}^{(0)}+\sqrt{\epsilon} V_{x z}^{(1)}\right)\right. \\
& \left.+\sqrt{\epsilon} \rho_{s y} \alpha(y) V_{x y}^{(2)}\right] \frac{1}{f(y) x V_{x x}^{(0)}}\left(1-\sqrt{\epsilon} \frac{V_{x x}^{(1)}}{V_{x x}^{(0)}}\right)+\cdots \\
= & \left(\lambda(y)+\rho_{s z} \sigma B(t)\right) \frac{R(t, x)}{f(y) x} \\
& +\frac{\sqrt{\epsilon}}{f(y) x V_{x}^{(0)}}\left[\left(\lambda(y)+\rho_{s z} \sigma B(t)\right) D_{2} V^{(1)}+\lambda(y) D_{1} V^{(1)}\right. \\
& +\rho_{s z} \sigma D_{1} V_{z}^{(1)}-\rho_{s y} \alpha(y)\left(\phi^{\prime}(y)\right. \\
& \left.\left.+\rho_{s z} \sigma B(t) \psi^{\prime}(y)\right) D_{1}^{2} V^{(0)}\right]+\cdots
\end{aligned}
$$

Here, we have used the fact that $V_{z}^{(0)}=B(t) V^{(0)}$. Using the explicit expressions of $V^{(0)}$ and $V^{(1)}$ given respectively in Theorem 2 and Theorem 4 and the fact that $D_{2} V^{(0)}=-D_{1} V^{(0)}$, we have the following asymptotic result for the optimal strategy. 
Theorem 5. The first order correction of the optimal strategy $\pi^{*}(t, x, y, z)$ is

$$
\tilde{\pi}^{*}=\pi^{*(0)}+\sqrt{\epsilon} \pi^{*(1)}
$$

where

$$
\begin{aligned}
& \pi^{*(0)}=\frac{1}{q f(y)}\left(\lambda(y)+\rho_{s z} \sigma B(t)\right)\left(\frac{q}{1-p}+\frac{\eta e^{r(t-T)}}{x}\right), \\
& \pi^{*(1)}=\frac{1}{q f(y)}\left(\rho_{s z} \sigma g_{2}(t)-\Gamma \rho_{s y} \alpha(y)\left(\phi^{\prime}(y)+\rho_{s z} \sigma B(t) \psi^{\prime}(y)\right)\right)\left(\frac{q}{1-p}+\frac{\eta e^{r(t-T)}}{x}\right) .
\end{aligned}
$$

Now, we claim that the leading order term of the optimal strategy can recover the optimal value function up to the first order correction as given in following theorem.

Theorem 6. The suboptimal strategy $\pi^{*(0)}$ given by $(75)$ recovers the approximation up to the first order,

$$
V^{(0)}+\sqrt{\epsilon} V^{(1)}
$$

of the optimal value function $V^{(\epsilon)}(t, x, y, z)$ given in Section 3. More specifically, if we denote $V^{\epsilon, \pi^{*(0)}}$ the value function corresponding to the strategy $\pi^{*(0)}$ and assume that it has expansion

$$
V^{\epsilon, \pi^{*(0)}}=V^{\pi^{*(0)},(0)}+\sqrt{\epsilon} V^{\pi^{*(0)},(1)}+\epsilon V^{\pi^{*(0)},(2)}+\cdots,
$$

then it follows that

$$
V^{\pi^{*(0)},(0)}=V^{(0)}, \text { and } V^{\pi^{*(0)},(1)}=V^{(1)},
$$

where $V^{(0)}$ and $V^{(1)}$ are defined in Theorem 2 and Theorem 4, respectively.

Proof. See Appendix C.

\section{CONCLUSION}

In this paper, we studied the optimization problem under an extended Heston model in which a fast mean-reverting volatility factor is added on top of the CIR process that drives the volatility in the pure Heston stochastic volatility model. Using asymptotic analysis, we were able to derive explicit approximations for the optimal value function and the optimal investment strategy up to the first order correction term for the HARA utility functions. This study extends the results of [5] in the sense that the Heston model is brought into a multi-scale model. It also extends the results of [4] as the Black-Scholes model is replaced by the Heston model in the case of the HARA utility functions. 
The accuracy of our asymptotic approximation will be treated in a future work, which is in preparation. Another research plan is to consider the same problem under a more general model in which one additional slow varying factor of volatility is incorporated to the current market model.

\section{A. A PORTFOLIO PROBLEM UNDER HESTON MODEL}

In order to continue our asymptotic analysis in Section 3, we recall some basic results of the portfolio problem under the Heston stochastic volatility model, which is the case that $f(y)=1$ and $\mu(y, z)=\mu(z)$ is independent of $y$ in our model (2)-(4), and was studied by [5] for the case of power utility functions. Here, we consider the HARA utility functions $U(x)$ defined in (8), which also cover the case of power and exponential utility functions.

In this case, the optimal strategy, denoted by $\pi_{H}^{*}(t, x, z)$, is given by

$$
\pi_{H}^{*}(t, x, z)=-\frac{\lambda V_{x}^{H}+\rho_{s z} \sigma V_{x z}^{H}}{x V_{x x}^{H}},
$$

where $V^{H}(t, x, z)$ is the corresponding optimal value function satisfying the following PDE

$$
\begin{array}{r}
V_{t}^{H}+r x V_{x}^{H}+\kappa(\theta-z) V_{z}^{H}+\frac{1}{2} \sigma^{2} z V_{z z}^{H}-\frac{z\left(\lambda V_{x}^{H}+\rho_{s z} \sigma V_{x z}^{H}\right)^{2}}{2 V_{x x}^{H}}=0 \\
V^{H}(T, x, z)=U(x) .
\end{array}
$$

We suppose that the solution $V^{H}$ to the nonlinear PDE problem (77)-(78) is of the form

$$
V^{H}(t, x, z)=\frac{1-p}{p q}\left(\frac{q}{1-p} x a(t)+\eta\right)^{p} h(t, z),
$$

with terminal conditions $a(T)=1$ and $h(T, z)=1$. Substituting this solution into (77) yields

$$
\begin{array}{r}
\frac{1-p}{p q}\left(\frac{q}{1-p} x a(t)+\eta\right)^{p}\left(h_{t}+\kappa(\theta-z) h_{z}+\frac{1}{2} \sigma^{2} z h_{z z}+\frac{p}{1-p} \frac{z\left(\lambda h+\rho_{s z} \sigma h_{z}\right)^{2}}{2 h}\right) \\
+\left(\frac{q}{1-p} x a(t)+\eta\right)^{p-1}\left(a_{t}+r a(t)\right) x h=0 .
\end{array}
$$

We can split this equation into two equations

$$
\begin{aligned}
a_{t}+r a(t) & =0, \\
h_{t}+\kappa(\theta-z) h_{z}+\frac{1}{2} \sigma^{2} z h_{z z}+\frac{p}{1-p} \frac{z\left(\lambda h+\rho_{s z} \sigma h_{z}\right)^{2}}{2 h} & =0 .
\end{aligned}
$$


Taking into account the terminal condition $a(T)=1$, the solution of (80) is given by

$$
a(t)=e^{r(T-t)} .
$$

We assume that the PDE (81) with terminal condition $h(T, z)=1$ has a solution of the form

$$
h(t, z)=e^{A(t)+z B(t)},
$$

where $A(T)=0$ and $B(T)=0$. Plugging (82) into (81) leads to

$$
A_{t}+B_{t} z+\kappa(\theta-z) B(t)+\frac{1}{2} \sigma^{2} z B^{2}(t)+\frac{p}{1-p} \frac{z\left(\lambda+\rho_{s z} \sigma B(t)\right)^{2}}{2}=0 .
$$

This equation is separable, and we can split it into the following ODEs

$$
\begin{aligned}
A_{t}+\kappa \theta B(t) & =0 \\
A(T) & =0 \\
B_{t}+\frac{\sigma^{2}}{2}\left(1+\frac{p}{1-p} \rho_{s z}^{2}\right) B^{2}(t)+\left(\frac{p}{1-p} \rho_{s z} \sigma \lambda-\kappa\right) B(t)+\frac{p \lambda^{2}}{2(1-p)} & =0 \\
B(T) & =0 .
\end{aligned}
$$

Note that $\frac{\sigma^{2}}{2}\left(1+\frac{p}{1-p} \rho_{s z}^{2}\right)>0$ since $p<1$. Then, the equations (85)-(86) can be solved explicitly as follows. Let

$$
\begin{aligned}
\Delta_{H} & =\kappa^{2}-\frac{p}{1-p}\left(2 \kappa \rho_{s z} \sigma \lambda+\sigma^{2} \lambda^{2}\right) \\
g_{H} & =\frac{\kappa-\frac{p}{1-p} \rho_{s z} \sigma \lambda+\sqrt{\Delta_{H}}}{\kappa-\frac{p}{1-p} \rho_{s z} \sigma \lambda-\sqrt{\Delta_{H}}} \\
K_{H} & =\frac{1}{2}\left(\kappa-\frac{p}{1-p} \rho_{s z} \sigma \lambda\right) .
\end{aligned}
$$

Then, we have

- Case 1: $\Delta_{H}>0\left(\right.$ or, $\left.\lambda<\frac{\kappa}{\sigma}\left(-\rho_{s z}+\sqrt{\rho_{s z}^{2}+\frac{1-p}{p}}\right)\right)$.

$$
\begin{aligned}
A(t)= & \frac{\kappa \theta}{\sigma^{2}\left(1+\frac{p}{1-p} \rho_{s z}^{2}\right)}\left(\left(\kappa-\frac{p}{1-p} \rho_{s z} \sigma \lambda+\sqrt{\Delta_{H}}\right)(T-t)\right. \\
& \left.-2 \ln \left(\frac{1-g_{H} e^{(T-t) \sqrt{\Delta_{H}}}}{1-g_{H}}\right)\right) \\
B(t)= & \frac{\kappa-\frac{p}{1-p} \rho_{s z} \sigma \lambda+\sqrt{\Delta_{H}}}{\sigma^{2}\left(1+\frac{p}{1-p} \rho_{s z}^{2}\right)}\left(\frac{1-e^{\sqrt{\Delta_{H}}(T-t)}}{1-g_{H} e^{\sqrt{\Delta_{H}}(T-t)}}\right) .
\end{aligned}
$$


- Case 2: $\Delta_{H}=0\left(\right.$ or, $\left.\lambda=\frac{\kappa}{\sigma}\left(-\rho_{s z}+\sqrt{\rho_{s z}^{2}+\frac{1-p}{p}}\right)\right)$ and $T K_{H}+1>0$.

$$
\begin{aligned}
A(t)= & \frac{\kappa \theta}{\sigma^{2}\left(1+\frac{p}{1-p} \rho_{s z}^{2}\right)}\left(\left(\kappa-\frac{p}{1-p} \rho_{s z} \sigma \lambda\right)(T-t)\right. \\
& \left.-2 \ln \left(1+\frac{1}{2}\left(\kappa-\frac{p}{1-p} \rho_{s z} \sigma \lambda\right)(T-t)\right)\right), \\
B(t)= & \frac{\left(\kappa-\frac{p}{1-p} \rho_{s z} \sigma \lambda\right)^{2}}{2 \sigma^{2}\left(1+\frac{p}{1-p} \rho_{s z}^{2}\right)}\left(\frac{T-t}{1+\frac{1}{2}\left(\kappa-\frac{p}{1-p} \rho_{s z} \sigma \lambda\right)(T-t)}\right) .
\end{aligned}
$$

Therefore, the optimal value is given by

$$
V^{H}(t, x, z)=\frac{1-p}{p q}\left(\frac{q}{1-p} x a(t)+\eta\right)^{p} e^{A(t)+z B(t)},
$$

where the functions $A(t)$ and $B(t)$ are defined as above cases. Then, from (76) the optimal strategy $\pi_{H}^{*}$ is given by

$$
\pi_{H}^{*}=\frac{\left(\lambda+\rho_{s z} \sigma B(t)\right)\left(\frac{q}{1-p} x e^{r(T-t)}+\eta\right)}{q x e^{r(T-t)}} .
$$

Observe that as $p \rightarrow-\infty$ and $\eta=1$, the HARA utility function $U(x)$ in (8) converges to an exponential (CARA) utility function

$$
U_{\exp }(x)=-\frac{1}{q} e^{-q x}, q>0
$$

In this case, we suppose the solution for (77) is of the form

$$
V^{H}(t, x, z)=-\frac{1}{q} e^{-q x a(t)} h(t, z) .
$$

Then, direct substitution leads to

$$
\begin{aligned}
a_{t}+r a & =0, \\
h_{t}+\kappa(\theta-z) h_{z}+\frac{1}{2} \sigma^{2} z h_{z z}-\frac{z\left(\lambda h+\rho_{s z} \sigma h_{z}\right)^{2}}{2 h} & =0 .
\end{aligned}
$$

Observe that (87) is the limit of (81) as $p \rightarrow-\infty$. Therefore, all formulas above for HARA utility functions are also valid for the exponential utility functions when letting $p \rightarrow-\infty$. 


\section{B. PROOF OF THEOREM 6}

Now, we try to find the solution $V^{(1)}$ of the PDE (41) with the terminal condition (49) of the form

$$
V^{(1)}(t, x, z)=\left(\kappa \theta g_{1}(t)+g_{2}(t) z\right) V^{(0)}(t, x, z)
$$

with $g_{1}(T)=0$ and $g_{2}(T)=0$. Substituting (88) into (41) and using (29) and (33) yields

$$
\begin{gathered}
\kappa \theta g_{1}^{\prime}+z g_{2}^{\prime}+\kappa(\theta-z) g_{2}(t)+\sigma^{2} z B(t) g_{2}(t)+\rho_{s z} \sigma \Gamma\left(\bar{\lambda}+\rho_{s z} \sigma B(t)\right) z g_{2}(t)= \\
z\left(\rho_{s y} \Gamma^{2} F_{3}+\sigma \Gamma\left(\rho_{y z} F_{1}+\rho_{s y} \rho_{s z} \Gamma\left(F_{1}+F_{4}\right)\right) B(t)+\rho_{s z} \sigma^{2} \Gamma\left(\rho_{y z}+\rho_{s y} \rho_{s z} \Gamma\right) F_{2} B^{2}(t)\right)
\end{gathered}
$$

where $B(t)$ is defined as in Theorem 2 .

Therefore, (89) is separable in $z$ and we can split it into two ODEs

$$
\begin{aligned}
g_{1}^{\prime}(t) & =-g_{2}(t), \\
g_{2}^{\prime}(t)+a(t) g_{2}(t) & =b(t),
\end{aligned}
$$

where

$$
\begin{aligned}
a(t)= & \sigma^{2}\left(1+\rho_{s z}^{2} \Gamma\right) B(t)+\left(\rho_{s z} \sigma \bar{\lambda} \Gamma-\kappa\right) \\
b(t)= & \rho_{s y} \Gamma^{2} F_{3}+\sigma \Gamma\left(\rho_{y z} F_{1}+\rho_{s y} \rho_{s z} \Gamma\left(F_{1}+F_{4}\right)\right) B(t) \\
& +\rho_{s z} \sigma^{2} \Gamma\left(\rho_{y z}+\rho_{s y} \rho_{s z} \Gamma\right) F_{2} B^{2}(t) .
\end{aligned}
$$

Note that the ODE (91) with terminal condition $g_{2}(T)=0$ is a Riccati equation with constant coefficients, which can be solved explicitly using the integral factor method. Let $w(t)$ be the integral factor defined by

$$
w(t)=e^{-\int_{t}^{T} a(s) d s},
$$

which leads to

$$
g_{2}(t)=-\int_{t}^{T} \frac{w(s)}{w(t)} b(s) d s .
$$

Therefore, we study in two cases as follows:

- Case 1: $\Delta>0$.

By making use of (92), (83) and (24), direct computation gives

$$
\frac{w(s)}{w(t)}=e^{\sqrt{\Delta}(s-t)+2 \ln \frac{1-g e^{\sqrt{\Delta}(T-s)}}{1-g e^{\sqrt{\Delta}(T-t)}}} .
$$


By writing $b(t)$ in $(93)$ as

$$
b(t)=c_{0}+c_{1}\left(\frac{1-e^{\sqrt{\Delta}(T-t)}}{1-g e^{\sqrt{\Delta}(T-t)}}\right)+c_{2}\left(\frac{1-e^{\sqrt{\Delta}(T-t)}}{1-g e^{\sqrt{\Delta}(T-t)}}\right)^{2},
$$

with $c_{0}, c_{1}$ and $c_{2}$ given by (59), (60) and (61) respectively, it follows from (94) and (90) that

$$
\begin{aligned}
& g_{2}(t)=-\left(c_{0} I_{0}(t)+c_{1} I_{1}(t)+c_{2} I_{2}(t)\right), \\
& g_{1}(t)=-\left(c_{0} J_{0}(t)+c_{1} J_{1}(t)+c_{2} J_{2}(t)\right),
\end{aligned}
$$

where the functions $I_{0}(t), I_{1}(t), I_{2}(t), J_{0}(t), J_{1}(t)$ and $J_{2}(t)$ are defined by

$$
\begin{aligned}
& I_{0}(t)=\int_{t}^{T} e^{\sqrt{\Delta}(s-t)}\left(\frac{1-g e^{\sqrt{\Delta}(T-s)}}{1-g e^{\sqrt{\Delta}(T-t)}}\right)^{2} d s, \\
& I_{1}(t)=\int_{t}^{T} e^{\sqrt{\Delta}(s-t)} \frac{\left(1-g e^{\sqrt{\Delta}(T-s)}\right)\left(1-e^{\sqrt{\Delta}(T-s)}\right)}{\left(1-g e^{\sqrt{\Delta}(T-t)}\right)^{2}} d s, \\
& I_{2}(t)=\int_{t}^{T} e^{\sqrt{\Delta}(s-t)}\left(\frac{1-e^{\sqrt{\Delta}(T-s)}}{1-g e^{\sqrt{\Delta}(T-t)}}\right)^{2} d s, \\
& J_{i}(t)=\int_{t}^{T} I_{i}(s) d s, \quad i=0,1,2 .
\end{aligned}
$$

By direct computation, we can easily see that $I_{0}(t), I_{1}(t), I_{2}(t), J_{0}(t), J_{1}(t)$ and $J_{2}(t)$ are explicitly given by (53), (54), (55), (56), (57) and (58) respectively. Therefore, $g_{2}(t)$ and $g_{1}(t)$ are given by (52) and (51), respectively.

- Case 2: $\Delta=0$ and $T K+1>0$.

Similar to Case 1, a series of straightforward calculations leads to

$$
\frac{w(s)}{w(t)}=e^{2 \ln \frac{1+\frac{1}{2}\left(\kappa-\rho_{s z} \sigma \bar{\lambda} \Gamma\right)(T-s)}{1+\frac{1}{2}\left(\kappa-\rho_{s z} \sigma \bar{\lambda} \Gamma\right)(T-t)}} .
$$

Then (94) is equivalent to

$$
g_{2}(t)=-\int_{t}^{T}\left(\frac{1+K(T-s)}{1+K(T-t)}\right)^{2} b(s) d s
$$

where $K=\frac{1}{2}\left(\kappa-\Gamma \rho_{s z} \sigma \bar{\lambda}\right)$. We can write $b(t)$ in (93) as

$$
b(t)=\bar{c}_{0}+\bar{c}_{1} \frac{T-t}{1+K(T-t)}+\bar{c}_{2}\left(\frac{T-t}{1+K(T-t)}\right)^{2},
$$


where the constants $\bar{c}_{0}, \bar{c}_{1}$ and $\bar{c}_{2}$ are defined by (70),(71) and (72), respectively. Then, it follows from (97) and (90) that

$$
\begin{aligned}
& g_{2}(t)=-\left(\bar{c}_{0} \bar{I}_{0}(t)+\bar{c}_{1} \bar{I}_{1}(t)+\bar{c}_{2} \bar{I}_{2}(t)\right), \\
& g_{1}(t)=-\left(\bar{c}_{0} \bar{J}_{0}(t)+\bar{c}_{1} \bar{J}_{1}(t)+\bar{c}_{2} \bar{J}_{2}(t)\right),
\end{aligned}
$$

where the functions $\bar{I}_{0}, \bar{I}_{1}, \bar{I}_{2}(t), \bar{J}_{0}(t), \bar{J}_{1}(t)$ and $\bar{J}_{2}$ are defined by

$$
\begin{aligned}
& \bar{I}_{0}(t)=\int_{t}^{T}\left(\frac{1+K(T-s)}{1+K(T-t)}\right)^{2} d s, \\
& \bar{I}_{1}(t)=\int_{t}^{T} \frac{(1+K(T-s))(T-s)}{(1+K(T-t))^{2}} d s, \\
& \bar{I}_{2}(t)=\int_{t}^{T}\left(\frac{T-s}{1+K(T-t)}\right)^{2} d s, \\
& \bar{J}_{i}(t)=\int_{t}^{T} \bar{I}_{i}(s) d s, \quad i=0,1,2 .
\end{aligned}
$$

Again, direct computation shows that $\bar{I}_{0}(t), \bar{I}_{1}(t), \bar{I}_{2}(t), \bar{J}_{0}(t), \bar{J}_{1}(t)$ and $\bar{J}_{2}(t)$ are explicitly given by $(64),(65),(66),(67),(68)$ and (69), respectively. Therefore, it follows that $g_{2}(t)$ and $g_{1}(t)$ are given by $(63)$ and $(62)$, respectively.

\section{PROOF OF THEOREM ??}

To simplify the symbol, we denote $\widetilde{V}=V^{\epsilon, \pi^{*(0)}}$ and write its expansion as

$$
\widetilde{V}=\widetilde{V}^{(0)}+\sqrt{\epsilon} \widetilde{V}^{(1)}+\epsilon \widetilde{V}^{(2)}+\cdots .
$$

Then, $\widetilde{V}$ satisfies the following linear PDE

$$
\begin{array}{r}
\widetilde{V}_{t}+r x \widetilde{V}_{x}+\frac{z}{\epsilon} \mathcal{L}_{0} \widetilde{V}+\kappa(\theta-z) \widetilde{V}_{z}+\frac{1}{2} \sigma^{2} z \widetilde{V}_{z z} \\
+\frac{1}{\sqrt{\epsilon}} \rho_{y z} \sigma \alpha(y) z \widetilde{V}_{y z}+\frac{1}{2}\left(\pi^{*(0)}\right)^{2} f^{2}(y) z x^{2} \widetilde{V}_{x x} \\
+\pi^{*(0)} z x\left(\lambda(y) \widetilde{V}_{x}+\rho_{s z} \sigma f(y) \widetilde{V}_{x z}+\frac{1}{\sqrt{\epsilon}} \rho_{s y} f(y) \alpha(y) \widetilde{V}_{x y}\right)=0,
\end{array}
$$

with the terminal condition

$$
\widetilde{V}(T, x, y, z)=U(x) .
$$

Note that PDE (101) is (10) in which $\pi^{*(0)}$ is chosen as the strategy. This holds since, by definition, $\widetilde{V}$ is the value function associated with the strategy $\pi^{*(0)}$. Using $(75)$, 
we can rewrite (101) as

$$
\frac{z}{\epsilon} \mathcal{L}_{0} \widetilde{V}+\mathcal{L}_{t, x, z}(\lambda(y), \lambda(y)) \widetilde{V}+\frac{z}{\sqrt{\epsilon}} \mathcal{L}_{x, y, z} \widetilde{V}=0
$$

where the operators $\mathcal{L}_{t, x, z}\left(\lambda_{1}, \lambda_{2}\right)$ and $\mathcal{L}_{x, y, z}$ are defined by (32) and (39), respectively. Substituting the expansion (100) in (103) and collecting successive powers of $\sqrt{\epsilon}$, the terms in $\epsilon^{-1}$ lead to

$$
\mathcal{L}_{0} \widetilde{V}^{(0)}=0
$$

Then we choose $\widetilde{V}^{(0)}$ to be independent of $y$. The terms in $\epsilon^{-\frac{1}{2}}$ give

$$
\mathcal{L}_{0} \widetilde{V}^{(1)}=0
$$

due to the $y$-independence of $\widetilde{V}^{(0)}$. Similarly, $\widetilde{V}^{(1)}$ is independent of $y$. At the constant terms in $\epsilon$, we have

$$
\mathcal{L}_{0} \widetilde{V}^{(2)}+\mathcal{L}_{t, x, z}(\lambda(y), \lambda(y)) \widetilde{V}^{(0)}=0
$$

This is a Poisson equation for $\widetilde{V}^{(2)}$ in $y$, whose centering condition requires that

$$
\left\langle\mathcal{L}_{t, x, z}(\lambda(y), \lambda(y)) \tilde{V}^{(0)}\right\rangle=0
$$

Since $\widetilde{V}^{(0)}$ does not depend on $y$, it follows that

$$
\mathcal{L}_{t, x, z}(\tilde{\lambda}, \bar{\lambda}) \widetilde{V}^{(0)}=0
$$

Expanding the terminal condition (102), we obtain

$$
\widetilde{V}^{(0)}(T, x, z)=U(x) .
$$

Then we have $\widetilde{V}^{(0)}=V^{(0)}$.

The terms in $\sqrt{\epsilon}$ give

$$
z \mathcal{L}_{0} \widetilde{V}^{(3)}+\mathcal{L}_{t, x, z}(\lambda(y), \lambda(y)) \widetilde{V}^{(1)}+z \mathcal{L}_{x, y, z} \widetilde{V}^{(2)}=0
$$

Viewing (108) as a Poisson equation for $\widetilde{V}^{(3)}$ in $y$, the centering condition is given by

$$
\left\langle\mathcal{L}_{t, x, z}(\lambda(y), \lambda(y)) \widetilde{V}^{(1)}+z \mathcal{L}_{x, y, z} \widetilde{V}^{(2)}\right\rangle=0 .
$$

Since $\widetilde{V}^{(1)}$ is independent of $y$, it follows that

$$
\mathcal{L}_{t, x, z}(\tilde{\lambda}, \bar{\lambda}) \widetilde{V}^{(1)}=-z\left\langle\mathcal{L}_{x, y, z} \widetilde{V}^{(2)}\right\rangle .
$$

From (106) and (107), we have

$$
\mathcal{L}_{0} \widetilde{V}^{(2)}=-\frac{1}{z}\left(\mathcal{L}_{t, x, z}(\lambda(y), \lambda(y))-\mathcal{L}_{t, x, z}(\tilde{\lambda}, \bar{\lambda})\right) \widetilde{V}^{(0)}
$$




$$
=-\frac{1}{z}\left(\mathcal{L}_{t, x, z}(\lambda(y), \lambda(y))-\mathcal{L}_{t, x, z}(\tilde{\lambda}, \bar{\lambda})\right) V^{(0)} .
$$

This is the Poisson equation (35), so we can choose $\widetilde{V}^{(2)}(t, x, y, z)=V^{(2)}(t, x, y, z)+$ $c(t, x, z)$, for some function $c$ independent of $y$. When applying $\mathcal{L}_{x, y, z}$ on $V^{(2)}, c$ does not play a role and then the equation (109) is exactly the equation (40), which implies that $\widetilde{V}^{(1)}=V^{(1)}$. This completes the proof.

\section{ACKNOWLEDGMENTS}

The research of J.-H. Yoon was supported by a National Research Foundation of Korea grant funded by the Korean government (NRF-2016R1C1B2014017), and the National Research Foundation of Korea(NRF) Grant funded by the Korean Government (MSIP) (NRF-2017R1A5A1015722).

\section{REFERENCES}

[1] B. Øksendal, Stochastic Differential Equations: An Introduction with Applications, Universitext. Springer, Berlin (2003).

[2] G. Chacko, L.M. Viceira, Dynamic consumption and portfolio choice with stochastic volatility in incomplete markets, Rev. Finan. Stud., 18 (2005), 1369-1402.

[3] J.-P. Fouque, M.J. Lorig, A fast mean-reverting correction to Heston's stochastic volatility model, SIAM J. Financial Math., 2 (2011), 221-254.

[4] J.-P. Fouque, R. Sircar, T. Zariphopoulou, Portfolio optimization and stochastic volatility asymptotics, Math. Finance, 27 (2017), 704-745.

[5] H. Kraft, Optimal portfolios and Heston's stochastic volatility model: an explicit solution for power utility, Quant. Finance 5 (2005), 303-313.

[6] R.C. Merton, Lifetime portfolio selection under uncertainty: The continuoustime case, Rev. Econ. Stat., 51 (1969), 247-257.

[7] R.C. Merton, Optimum consumption and portfolio rules in a continuous-time model, J. Econ. Theory, 3 (1971), 373-413.

[8] T. Zariphopoulou, A solution approach to valuation with unhedgeable risks, Finance Stochastics, 5 (2001), 61-82. 